\title{
Termini in der technischen Übersetzung: Rettungsanker, Tyrannen - oder auch nur Wörter?
}

\author{
Ulrike Oster \\ Universitat Jaume I, Castelló
}

The understanding of 'term' in traditional terminology theory reduces the lexical problems of technical translation to a mere substitution of the source-text term by a target-text term. In translation studies however, a number of issues have been highlighted which are not covered by traditional terminology theory, e.g. cultural specificity or the importance of textual and pragmatic considerations. This paper first analyses how the new communication and cognition-oriented approaches to terminology account for these aspects of technical translation. Then it briefly presents results of a language-pair and domain-specific study which allows us to exemplify some of the issues that have been discussed and to reach some specific conclusions for the translator of this linguistic combination.

\section{Termini aus der Perspektive des Übersetzers und der Überset- zungswissenschaft*}

Wenn es um fachsprachliche Übersetzung geht, wird der terminologischen Komponente meist eine vorrangige Bedeutung zugeschrieben und der Bereich Technik ${ }^{1}$ bildet hier keine Ausnahme. Auch wenn in der Literatur zur technischen Übersetzung makrostrukturelle Aspekte wie Textsortenkonventionen (Göpferich 1993, 1995) oder Sprechakte (Gamero 2001) nun einen größeren Raum einnehmen, entsteht oft der Eindruck, dass die lexikalischen Probleme beim technischen Übersetzen schon dann gelöst sind, wenn der Übersetzer den entsprechenden Terminus in der anderen Sprache gefunden hat. Dahinter steht offenbar ein von Wüsters Allgemeiner Terminologielehre (ATL) geprägter Terminusbegriff, bei dem jeder Terminus möglichst eineindeutig (d. h. ohne Polysemie und ohne Synonymie) auf einen in einem übersprachlichen Begriffssystem lokalisierten Begriff verweist. Eine äquivalente Benennung in der Zielsprache referiert auf denselben Begriff. Für die Übersetzung bedeutet dies in den Worten Weissenhofers (1992: 322-323):

Der Translator geht bei der Übersetzung in der ersten Phase semasiologisch vor, d. h. von der ausgangssprachlichen Benennung zum Begriff als kognitiver Denk- und Wissenseinheit, in der zweiten Phase jedoch onomasiologisch, d. h. vom übersprachlichen Begriff zur zielsprachlichen Benennung.

Als logische Konsequenz dieser Auffassung wird in vielen Arbeiten zu Terminologie und technischer bzw. Fachübersetzung an erster Stelle dem 
Auffinden von Terminologie in der Zielsprache Aufmerksamkeit gewidmet (z. B. Wright \& Wright 1997). Existiert ein Begriff in der Zielsprache nicht, bzw. ist noch keine Benennung eingeführt, muss der Übersetzer quasi als Terminologe tätig werden und einen adäquaten Neologismus prägen (Arntz 1993: 15-16).

Andererseits tritt gerade in der Praxis des Übersetzens die Problematik einer solchen restriktiven Sichtweise zu Tage, was sich auch in der übersetzungswissenschaftlichen Literatur widerspiegelt. Ein besonders relevanter Bereich ist hier die Kulturspezifik auf verschiedenen Ebenen der Fachkommunikation. Neben zwischensprachlichen Unterschieden bei Konventionen einzelner Fachtextsorten stehen auch die technischen Fachsprachen (bzw. konkreter: Fachbenennungen) im Blickpunkt. Hier stellt Arntz (2001: 87) fest:

\begin{abstract}
Wenn wir nun technische Terminologie verschiedener Sprachen miteinander vergleichen, können wir [...] zwei gegenläufige Tendenzen feststellen [...]. Auf der einen Seite führt die internationale Kooperation, bei der vielfach eine Sprachgemeinschaft eine dominierende Rolle spielt, dazu, daß in großer Zahl Entlehnungen und Lehnübersetzungen in viele Sprachen Eingang finden [...]. Auf der anderen Seite entwickeln sich die einzelnen Sprachen - auch die technischen Fachsprachen - in nicht unerheblichem Maße weiterhin spontan nach ihren eigenen Gesetzen.
\end{abstract}

Aus diesem Grund unterstreicht beispielsweise Horn-Helf (1999: 301318) die Bedeutung, die Kenntnisse zu sprachenspezifischen Terminusbildungskonventionen für den Übersetzer haben. Sie bedauert allerdings, dass kontrastive Arbeiten zur speziellen Terminusbildung noch ausstehen.

Für den Bereich der Begriffssysteme hat vor allem Schmitt (1986, 1989, 1999) die Existenz von kulturellen Unterschieden mit vielfältigen Beispielen aus den verschiedensten technischen Bereichen belegt. Zwar ist sich auch die ATL der Existenz von Unterschieden in den Begriffssystemen verschiedener Sprachen durchaus bewusst. Aufgrund ihrer vorrangigen Zielsetzung (Normung zum Zweck der internationalen Kommunikation) ist die logische Konsequenz daraus jedoch lediglich die Notwendigkeit, abweichende Begriffssysteme $\mathrm{zu}$ vereinheitlichen. So sagen Felber \& Budin (1989: 92): "Für dieses Problem gibt es nur eine Lösung, nämlich die bewußte Angleichung der Begriffe und Begriffssysteme durch deren Vereinheitlichung."

Zum anderen wird beim Übersetzen die Bedeutung von textuellen und pragmatischen Faktoren besonders sichtbar. So weist Shreve (1997: 772-773) darauf hin, dass ein zielsprachliches Äquivalent nicht nur semantisch, sondern auch sprachlich und pragmatisch in den Zieltext passen muss:

[...] the specific meaning, usage, and appropriateness of a term is always conditioned by its textual environment. [...] we should always remember that 
the target text is the final arbiter of whether the result of our terminological research process "fits" or not. The "fit" between term and text is semantic $[\ldots]$, linguistic $[\ldots]$, pragmatic [...].

Auch Rogers (1999, 2000) weist auf die Gefahren der "look-it-up-andslot-it-in"-Vorgehensweise hin, die auf der Annahme basiert, dass wissenschaftliche und technische Termini geringeren Texteinflüssen ausgesetzt seien (2000: 104). An Beispielen aus fahrzeugtechnischen Texten zeigt sie sodann, wie im Text dynamische Beziehungen zwischen Termini entstehen, während die durch Wörterbücher oder Begriffssysteme vermittelte Sicht statisch ist. Dies lässt sich laut Rogers darauf zurückführen, dass der Textproduzent unterschiedliche Perspektiven auf den gleichen Sachverhalt einnehmen kann. Sie kommt daher zu dem Schluss, dass ein dynamischer und kreativer Umgang des Übersetzers mit den zielsprachlichen Termini vonnöten ist.

Eine weitere Frage, die vom übersetzungswissenschaftlichen Standpunkt diskutiert wird, ist die der Unterscheidung zwischen Terminus und Wort, bzw. ihrer Relevanz für das Übersetzen (so zum Beispiel Varantola 1992; Pearson 1998; Thelen 2002a, 2002b). In diesem Zusammenhang weist Kaiser-Cooke (1995) darauf hin, dass die den Termini traditionell zugeschriebenen Eigenschaften (präzise Definition, Bestandteil eines Systems) möglicherweise doch nicht so gut geeignet sind, um sie von allgemeinsprachlichen Wörtern zu unterscheiden. Denn einerseits entsprechen nicht alle Fachausdrücke im Text einer präzisen Definition und andererseits kann man von fast allen Begriffen sagen, dass sie Teil eines Systems sind. Sie kommt daher zu dem Schluss, dass andere Kriterien notwendig wären und fragt sich, ob es tatsächlich einen solchen grundlegenden Unterschied gibt zwischen den eigentlichen Fachwörtern und anderen textabhängigen und fachspezifischen Einheiten.

Nun gewinnen in der letzten Zeit in der Terminologie neue Ansätze immer mehr an Bedeutung, die gerade diese von Übersetzern eingeforderte kommunikative, textorientierte Perspektive berücksichtigen. Deshalb sollen im folgenden Abschnitt einige der Neuerungsansätze innerhalb der Terminologie kurz dargestellt und geprüft werden, inwieweit sie Antwort auf die angesprochenen Problembereiche geben.

\section{Neuere Ansätze zum Verständnis von Termini in der Terminologie}

In den letzten Jahrzehnten mehren sich die kritischen Stimmen bezüglich der strikten und idealisierenden Terminusauffassung der Allgemeinen Terminologielehre, gestützt auf empirische Befunde und neue Erkenntnisse aus der Linguistik und speziell der Fachsprachenforschung. So hat eine Reihe von Autoren unterschiedliche Aspekte der ATL kritisch untersucht (umfassend dargestellt zum Beispiel in Cabré 1999 oder Temmerman 2000). Hier beschränken wir uns auf einen kurzen Überblick 
über einige Autoren, deren Beiträge diese Kritikpunkte aufgreifen und die entweder innerhalb der Allgemeinen Terminologielehre Ergänzungen vorschlagen (Weissenhofer 1995; Gerzymisch-Arbogast 1996), oder den Anspruch erheben, einen alternativen oder zumindest komplementären Ansatz zu begründen (Cabré 1999, 2003; Temmerman 2000).

\subsection{Vorschläge im Rahmen der Allgemeinen Terminologielehre}

Weissenhofer (1995) stellt fest, dass Wüsters Wortmodell (Wüster 1959/60) nicht in der Lage ist, eine Erklärung für kontextuelle Aspekte sowie für Polysemie und Synonymie zu bieten, die Rolle der Beziehungen von Termini zu anderen Zeichen zu berücksichtigen oder die Ebenen von langue und parole ausreichend zu unterscheiden. Deshalb schlägt er ein modifiziertes Modell vor, das außerdem Vagheit und Prototypizität der Begriffe in Betracht zieht.

Einen ähnlichen Ansatz verfolgt Gerzymisch-Arbogast (1996). Auch sie ergänzt Wüsters Modell um eine Verwendungsebene, das so zu einem kontextspezifischen Terminusmodell wird. Das Prinzip der Eineindeutigkeit der ATL wird hier also nicht abgelehnt, sondern nur auf die Systemebene beschränkt. Beim Übergang des Terminus vom System auf die Verwendungsebene (den Text) können eine Reihe von sogenannten Kontaminationen auftreten. Das bedeutet, dass Termini im Text anders verwendet werden können als dies ihre Stellung im Begriffssystem vorschreiben würde. In Bezug auf die Benennung unterscheidet Gerzymisch-Arbogast die Similaritäts-, Inklusions-, Subordinations-, Intersektions- und Metonymiekontamination. Bezüglich des Begriffs werden Aspektivierung, Terminologisierung, terminologische Polyvalenz sowie autorenspezifische Anwendung dargestellt. Um solche Kontaminationen zu erkennen schlägt Gerzymisch-Arbogast eine Analysemethode vor, bei der die Relationen des Terminus im Text mit jenen im System verglichen werden. Aufgrund dieser Analyse entscheidet der Übersetzer dann bei der Erstellung des Zieltextes, ob ein kontaminierter ausgangssprachlicher Terminus auch im Zieltext als kontaminierter Terminus oder aber unkontaminiert wiedergegeben wird.

Beide Modelle bewegen sich innerhalb der ATL, versuchen jedoch deren Terminusmodell $\mathrm{zu}$ erweitern und somit den tatsächlichen Gegebenheiten in der fachsprachlichen Kommunikation (sprich der Existenz von Polysemie und Synonymie sowie dem Einfluss von pragmatischen und textuellen Faktoren) anzupassen. Ein Unterschied scheint jedoch auf konnotativer Ebene zu bestehen. Während Weissenhofer Vagheit und Prototypizität neutral als natürliche Eigenschaften von Sprache darstellt, lässt die Bezeichnung "Kontamination" jedoch eine negative Wertung der textspezifischen Verwendungsvarianten zu. 


\subsection{Vorschläge für ein alternatives Verständnis der Terminologie}

Cabré und Temmerman dagegen gehen in ihren Beiträgen über eine bloße Modifikation des traditionellen Modells hinaus und versuchen neue Grundlagen für die terminologische Arbeit zu legen. Beide gehen von ähnlichen Voraussetzungen aus, da sie die genannten Unzulänglichkeiten der Allgemeinen Terminologielehre zu überwinden suchen, verwenden jedoch unterschiedliche theoretische Ansätze und Herangehensweisen, die verschiedene Aspekte in den Vordergrund stellen. Ihre Modelle sollen jetzt kurz skizziert werden, wobei es sicher nicht möglich ist, den Theorien insgesamt gerecht zu werden. Das Ziel unserer Ausführungen ist es deshalb auch nur, diejenigen Punkte darzustellen, die für unser Anliegen - das Terminusverständnis des technischen Übersetzers besonders relevant sind.

\subsubsection{Der kommunikative Ansatz}

Die von Cabré (1999) vorgeschlagene kommunikative Terminologielehre (teoría comunicativa de la terminología) setzt sich - wie ihr Name zum Ausdruck bringt - die Beschreibung terminologischer Einheiten in realer Kommunikation zum Ziel. Dabei werden alle Ebenen der Sprache (Grammatik, Semantik, Text) und des Diskurses (hoher, mittlerer und niedriger Grad der Spezialisierung) berücksichtigt. Die Existenz der Benennungsvariation wird als unvermeidliches Phänomen natürlicher Sprache ausdrücklich anerkannt.

Termini erfüllen zwei Funktionen: einerseits Fachwissen darzustellen/zu repräsentieren und andererseits Kommunikationsmittel für eben dieses Wissen zu sein. Aus dieser Doppelfunktion leiten sich laut Cabré (1999: 80-81) zwei Grundformen der terminologischen Arbeit ab. Terminologie, die sich auf die darstellende Funktion konzentriert, behandelt in vitro-Fachwissen (über das ein Konsens hergestellt wurde, d. h. das einer Normung unterzogen wurde) und kann präskriptiv eingesetzt werden. Sie dient zum Beispiel zu Zwecken der internationalen Terminologienormung. Terminologiearbeit jedoch, die die kommunikative Funktion in den Mittelpunkt stellt, beobachtet in-vivo-Fachwissen (das spontan, natürlich auftritt) und kann daher nur deskriptiv vorgehen. Eine der wichtigsten Funktionen dieser Form der Terminologie ist es, Hilfsmittel für das Übersetzen oder das Technical Writing zu erarbeiten.

Cabré versteht die kommunikative Terminologielehre als ein interdisziplinäres Wissensfeld, in dem Kognitions-, Kommunikations- und Sprachwissenschaft zusammenfließen. Terminologie muss daher auf jeweils allgemeinen Theorien über die folgenden drei Bereiche aufbauen:

- Kognition, d. h. darüber, wie Realität konzeptualisiert wird. Gegenstand dieser Theorie sind - vereinfacht gesagt - Begriffe (unidades de cono- 
cimiento), zu denen die Fachbegriffe (unidades de conocimiento especializado) als Gegenstand der Terminologie gehören;

- Kommunikation. $\mathrm{Zu}$ den von dieser Theorie behandelten Einheiten gehören auch die entsprechenden Einheiten der Fachkommunikation;

- Sprache. Auch hier stellen die von der Terminologie behandelten Einheiten (Termini) einen besonderen Fall der übrigen sprachlichen Zeichen dar.

Für den Terminusbegriff bedeutet dies, dass eine terminologische Einheit gleichzeitig kognitive, soziokulturelle und sprachliche Einheit ist. Als Einheiten der Sprache haben Termini prinzipiell die gleichen Eigenschaften wie andere lexikalische Einheiten. Dies bezieht sich zum Beispiel auf ihren referentiellen Charakter oder ihre Funktionen im Diskurs. Jede dieser Einheiten (terminologischer Art oder nicht) ist für einen Sprecher mit einer Vielzahl von Informationen grammatischer, pragmatischer und enzyklopädischer Natur verbunden. Per se, also außerhalb eines kommunikativen Kontextes sind solche lexikalischen Einheiten weder Wort noch Terminus. Erst durch den Gebrauch in einer fachlichen Kommunikationssituation aktiviert der Sprecher/Hörer fachspezifische Merkmale, bzw. blendet sie bei Verwendungen in anderen Kontexten aus (Cabré 1999: 103).

\subsubsection{Der soziokognitive Ansatz}

Temmerman (2000, 2002) schlägt für die terminologische Forschung einen soziokognitiven Ansatz vor. Dieser ist durch folgende, an der kognitiven Semantik orientierte Grundsätze gekennzeichnet, die den Prinzipien der Wiener Schule widersprechen:

- Der onomasiologischen Perspektive der Allgemeinen Terminologielehre wird ein semasiologischer Ansatz vorgezogen, weil es Begriffe gibt, die erst durch das Handeln und die Verstehensprozesse des Menschen zustande gekommen sind (Biotechnologie, Klonen).

- An Stelle von klar umrissenen Begriffen sieht Temmermans Ansatz Verstehenseinheiten (units of understanding) vor, die in den meisten Fällen flexible Kategorien mit Prototypenstruktur sind. Jede Kategorie wird als Teil eines kognitiven Modells (idealized cognitive model ICM) aufgefasst, das eine inter- und intrakategorielle Struktur besitzt, die jeder Sprecher für sich durch seinen Verstehensprozess aufbaut.

- Während die ATL knappe, intensionale Definitionen vorzieht, schlägt Temmerman "Muster" (templates) für die Bedeutungsbeschreibung vor, die mehr von jener Information aufnehmen, die traditionell als "enzyklopädisch" bezeichnet und aus der Definition ausgeschlossen wurde.

- Statt vom Prinzip der Eineindeutigkeit geht dieses Modell davon aus, dass Polysemie und Synonymie nicht nur möglich sind, sondern 
bestimmte Funktionen erfüllen und somit notwendig sind. Ein Grund für die Existenz (nahezu) synonymer Benennungen liegt darin, dass oft für einen Begriff mehrere mögliche Benennungsmotivationen vorliegen können (vgl. auch schon Drozd \& Seibicke 1973: 170). Durch die Wahl der einen oder der anderen Benennung hat der Sprecher die Möglichkeit bestimmte Bedeutungsnuancen auszudrücken: "Depending on which of the [...] elements a language user wants to stress in a particular context the use of one or another of the synonyms can be appropriate for more nuanced communication" (Temmerman 2000: 151).

- Im Gegensatz zur ATL, in der die synchronische Perspektive bevorzugt wird, führt schließlich die Erkenntnis, dass Kategorien sich verändern und Termini Bedeutungsverschiebungen durchlaufen, zu der Einsicht, dass gerade eine diachronische Sichtweise für die terminologische Forschung interessant ist.

Aufgrund der kognitiven Orientierung ihres Ansatzes interessiert sich Temmerman für die Benennung als Ausdruck eines Verstehensprozesses. Während dieses Prozesses wird ein neuer Begriff (eine Kategorie) mit einer bestehenden Struktur (einem kognitiven Modell) in Verbindung gebracht. Die Benennung spiegelt die vorgenommene Einordnung wider. Ein weiterer wichtiger Punkt ist dabei die Rolle, die konzeptuelle Metaphern in diesem Prozess des menschlichen Verstehens spielen (auch und gerade bei wissenschaftlichen und technischen Vorgängen) und wie diese Metaphern sich lexikalisch niederschlagen. Auch darin unterscheidet sich Temmermans Ansatz stark von der traditionellen Sicht, die anderen Benennungsformen den Vorrang vor metaphorischen Bildungen gibt.

\subsection{Fazit: Welche der genannten Aspekte sind im Hinblick auf das Terminusverständnis des technischen Übersetzers relevant?}

Die Bedeutung der Kommunikation. Gemeinsam ist allen genannten Ansätzen, dass der Akzent auf der Kommunikation bzw. der Verwendungsebene liegt. Der Übersetzer begegnet dem Terminus nicht im System (wenn denn ein solches angenommen wird), sondern im Text. Dort sind die Fachwörter einer nicht immer einheitlichen Verwendung unterworfen. Diese uneinheitliche Verwendung, pragmatisch und durch Textkonventionen bedingt, ist durch Gerzymisch-Arbogasts "Kontaminationen" oder die von Weissenhofer und Temmerman angenommene Flexibilität und Prototypenstruktur erklärbar. Bei der Erstellung des Zieltextes sind daher ebenfalls pragmatische und textuelle Konventionen zu beachten. Die neueren Terminusmodelle sind also kompatibel mit der seitens der Übersetzungswissenschaft geforderten pragmatischen Einpassung des Zielterminus in den Text.

Die Bedeutung der Kognition. Als ein weiterer wichtiger Punkt erscheint uns die Akzentuierung der kognitiven Vorgänge, die bei der 
Benennungsbildung eine Rolle spielen (Temmerman), also die Motivation der Benennungen, insbesondere der Rückgriff auf metaphorische Modelle. Im Bereich der Technik liegt hier mit Jakob (1991) bereits eine richtungsweisende Studie vor. Temmerman weist außerdem darauf hin, dass ein Teil dieser Modelle kulturspezifisch sein kann, was wiederum für die Übersetzung von Bedeutung ist: "Translators who are aware of the impact of metaphorical reasoning on lexicalisation in scientific language will develop the ability to distinguish between metaphorical models which are language and culture independent and those which are not" (Temmerman 2002: 211).

Temmermans Ansatz setzt die fachliche Benennungsbildung zu allgemeinen menschlichen Verstehensprozessen in Beziehung. Diese wiederum orientieren sich an kognitiven Modellen (ICMs), die schon per definitionem kulturspezifisch sind oder zumindest sein können (Lakoff 1987: 68-69). Die Erklärung der oben erwähnten Kulturspezifizität von Begriffssystemen und Konventionen der Benennungsbildung ist daher in einem solchen Terminologiemodell wesentlich einfacher als mit der traditionellen Sichtweise.

Die Unterscheidung von Terminus und Wort. Fachsprachliche Begriffe werden sowohl bei Cabré als auch bei Temmerman nicht grundsätzlich von allgemeinsprachlichen Konzepten unterschieden. Es handelt sich um Wissens- oder Verstehenseinheiten (unidades de conocimiento units of understanding), die Fachliches zum Inhalt haben. Die auf diese Begriffe verweisenden Benennungen werden erst in der entsprechenden (fachgebundenen) Kommunikationssituation zu Termini, indem ihre fachlichen Eigenschaften aktiviert werden (Cabré). Für das Erkennen der Fachlichkeit eines Terminus ist aber ein für kompetente Fachübersetzung ohnehin erforderliches $\mathrm{Maß}$ an Sachkenntnis notwendig. Ob man sich diese in Form des traditionellen Begriffssystems vorstellt, oder wie in Temmermans Ansatz als kognitives Modell, hat zwar bedeutende theoretische Implikationen, ist aber wohl für die Übersetzungspraxis weniger entscheidend, da es sich bei der Repräsentation des Übersetzers nur um eine Annäherung an das viel strukturiertere Expertenwissen handeln kann.

Die Bedeutung von Kommunikationssituation und Fachlichkeit für die Terminuserkennung ist zwar an sich nichts Neues, wichtig erscheint uns jedoch die Relativierung des konzeptuellen Unterschieds zwischen Wort und Terminus.

\section{Ergebnisse einer empirischen Untersuchung}

Nachdem die neueren Ansätze in der Terminologie uns allgemeine Antworten auf und Erklärungen für die in der Praxis des technischen Übersetzens festgestellten Diskrepanzen zur traditionellen Terminologielehre geliefert haben, wollen wir nun versuchen, anhand konkreter Daten eines bestimmten Sprachenpaars (Deutsch-Spanisch) und Fachgebiets (die 
Herstellung von keramischen Fliesen) zu genaueren Aussagen zu kommen, die für den Übersetzer dieser Sprachrichtung hilfreich sein könnten. Hierbei werden wir jeweils ein Beispiel bezüglich der Kommunikation (Terminusverwendung im Text) und der Kognition (sprachspezifische Konventionen bei der Fachwortbildung) erläutern.

Den Hintergrund hierfür liefert ein umfangreicher Vergleich von Parallelkorpora (dargestellt in Oster 2003, 2004a, 2004b). Darin werden zum einen die Form der Benennungen in beiden Sprachen untersucht und zum anderen die Art der semantischen Relation, die bei polylexematischen Benennungen zwischen den beiden unmittelbaren Terminuskonstituenten vorliegt. Ergänzend dazu haben wir für diese Arbeit die spanischen Entsprechungen der deutschen zusammengesetzten Benennungen in zwei- oder mehrsprachigen Nachschlagewerken recherchiert. $^{2}$

\subsection{Stilistische Konventionen: Erhöhung der Textkohäsion versus Redundanzvermeidung}

In den beiden Parallelkorpora zeigt sich ein deutlicher Unterschied bezüglich des Anteils von monolexematischen Benennungen, der im Spanischen mit 38,8 \% bedeutend höher liegt als im Deutschen $(22,4 \%)$. Diesen Unterschied kann man auf mehrere Ursachen zurückführen. Hierfür möchten wir auf eine Unterscheidung von Gómez (2001) zurückgreifen, die in einer Untersuchung zu spanischen Übersetzungsäquivalenten von deutschen Nominalkomposita zwischen lexikalischen (monolexemas del lexicón) und kontextuellen Monolexemen (monolexemas de contexto) differenziert. Erstere sind monolexematische spanische Entsprechungen eines deutschen lexikalisierten Kompositums. Ein Beispiel aus unserem Fachgebiet wäre Stufenplatte $\rightarrow$ peldaño. Hier kommt auch die im Spanischen wesentlich stärkere Tendenz zur Derivation zum Tragen (jabonera versus Seifenschale).

Kontextuelle monolexematische Benennungen im Sinne von Gómez sind dagegen das Ergebnis einer Übersetzungsstrategie, die auf die stilistischen Konventionen der Zielsprache baut. So führt Gómez u. a. folgende Beispiele an: Klimaprotokoll $\rightarrow$ protocolo, Regelungensituation $\rightarrow$ legislación.

Diese kontextuellen Simplizia werden entweder deshalb eingesetzt, weil - wie im ersten Fall - eine der Konstituenten schon im näheren Kontext vorhanden ist, oder - wie im zweiten Fall - weil der Inhalt der ausgelassenen Konstituente als redundant angesehen wird (Gómez 2001: 329). Man könnte diese Strategie der Übersetzungsrichtung Deutsch Spanisch also auf die Formel "Vermeide Redundanz" bringen.

Zum Teil lässt sich der zahlenmäßige Unterschied also einfach dadurch erklären, dass das Spanische eine stärkere Vorliebe für einfache und abgeleitete Wörter hat und das Deutsche dagegen gerne Komposita 
bildet. Da unser Korpus aber die Verhältnisse von Texten widerspiegelt, ist nicht auszuschließen, dass der Effekt teilweise auch auf kontextuelle Monolexeme zurückzuführen ist, besonders wenn man beachtet, dass eine ganze Reihe von Benennungen, denen im Parallelkorpus ausschließlich einfache Formen entsprechen, in den Fachwörterbüchern durch polylexematische Benennungen wiedergegeben werden, wie zum Beispiel:

\begin{tabular}{|c|c|c|c|}
\hline Becherwerk & $\rightarrow$ & $\begin{array}{l}\text { Korpus } \\
\text { cangilones }\end{array}$ & $\begin{array}{l}\text { Fachwörterbuch } \\
\text { elevador de cangilones }\end{array}$ \\
\hline Strangpresse & $\rightarrow$ & extrusionadora & prensa de extrusión, galletera de extrusión \\
\hline Transportband & $\rightarrow$ & cinta & cinta transportadora \\
\hline
\end{tabular}

An einem weiteren Beispiel soll dies etwas ausführlicher erläutert werden. Im deutschsprachigen Korpus sind mehrere zusammengesetzte Benennungen mit dem Determinatum Wagen $\mathrm{zu}$ finden (Brennwagen, Transportwagen, Tunnelofenwagen). Wagen allein (als theoretisch mögliche anaphorische Kürzung) kommt in keinem der Kontexte vor. Dieses ausschließliche Vorkommen der Langform kann mit einer der Hauptfunktionen deutscher Komposita in Beziehung gesetzt werden. So dient die Wiederholung von lexikalischen Elementen in Komposita (Isotopieketten, beschrieben zum Beispiel in Wladowa 1975, Schröder 1978, 1983, mit Bezug auf das Übersetzen auch bei Emsel 2001) der Verstärkung der Textkohäsion, wie in folgendem Beispiel aus unserem Korpus deutlich wird:

Beim Brennen ist es wichtig, daß die Ware nicht einer zu plötzlichen Temperaturerhöhung ausgesetzt wird. Die mit Fliesen vollgesetzten Brennwagen werden deshalb zunächst in einen Trockenkanal gefahren, [...]. Die Ausgangstemperatur des Trockenkanals - etwa $130^{\circ} \mathrm{C}$ - ist gleichzeitig die Eingangstemperatur für den $\underline{\text { Brennofen. }}$

Speziell für die deutsche Sprache der Technik hat Dederding (1982, 1983) diesen Effekt als (gewünschte) "Erhöhung der Redundanz" beschrieben, was der für das Spanische festgestellten stilistischen Tendenz genau entgegenläuft. Unsere Belege sprechen dafür, dass die Regel der Redundanzvermeidung im Spanischen auch für technische Texte gilt. So gibt das Fachwörterbuch als Entsprechung für Tunnelofenwagen zwar vagoneta de horno túnel an, in den Texten erscheint jedoch durchgehend nur vagoneta. Als Grund ist hier anzunehmen, dass die Angabe einer Funktion (Brennen, Transport, Tunnelofen, etc.) als nicht notwendig erachtet wird, da sie aus dem Kontext klar hervorgeht. Hinzu kommt, dass spanische Mehrwortbenennungen aufgrund der möglichen syntaktischen Modifikationen (beispielsweise durch Einfügen eines bestimmten Artikels: carga de molde / carga del molde) an sich instabiler sind, ihre Einheit also auch leichter aufgebrochen werden kann.

In Bezug auf die Behandlung von kürzeren oder längeren Termvarianten des Ausgangstexts plädiert Sager (1994) für den Einsatz von 
entsprechenden Varianten im Zieltext, wenn es in der Zielsprache für eine ähnliche Kontextsituation auch eine entsprechende rhetorische oder stilistische Konvention gibt. Im Fall von divergierenden Textkonventionen wie den oben beschriebenen könnte man hinzufügen, dass ein aktiver und bewusster Umgang mit Varianten erforderlich ist. Das heißt, man sollte nicht nur dann an Varianten denken, wenn im Ausgangstext eine Variante anzutreffen ist. Denn aus pragmatischen oder stilistischen Gründen kann auch dann eine kürzere Variante notwendig sein, wenn im Ausgangstext die Standardvariante steht (oder eben umgekehrt). Für die hier untersuchte Sprachenkombination und Fachrichtung könnte man für die Übersetzungsrichtung Deutsch $\rightarrow$ Spanisch die Redundanzvermeidung, sowie für die entgegengesetzte Richtung Spanisch $\rightarrow$ Deutsch die Stärkung der Textkohäsion anstreben.

\subsection{Konventionen bei der Fachwortbildung}

Eines der Ergebnisse der oben genannten Studie ist, dass die Finalrelation (wie in Beschichtungsanlage oder Bindemittel) in den komplexen substantivischen Benennungen der deutschen Fachsprache der Keramikherstellung besonders häufig vorkommt. Nehmen wir nun den Fall, dass für eine deutsche komplexe Benennung mit Finalrelation in den Nachschlagewerken kein äquivalenter Terminus zu finden ist. Aufgrund pragmatischer Überlegungen entscheidet sich der Übersetzer, die Motivation der deutschen Benennung beizubehalten. Während aber im Deutschen das Kompositum die bei weitem vorherrschende Form der komplexen Benennungen ist, kann Finalität im Spanischen auf verschiedene Arten ausgedrückt werden, über die folgende Tabelle einen Überblick gibt.

\begin{tabular}{|c|c|c|c|}
\hline Bildungsmodell & $\begin{array}{l}\text { Anzahl der } \\
\text { Belege }\end{array}$ & Beispiel Spanisch & deutsche Entsprechung \\
\hline$N+$ Suffix $^{3}$ & 41 & $\begin{array}{l}\text { plegadora } \\
\text { cortador }\end{array}$ & $\begin{array}{l}\text { Blechbiegemaschine } \\
\text { Abschneidevorrichtung }\end{array}$ \\
\hline $\mathrm{N}+\mathrm{de}+\mathrm{N}$ & 72 & balsa de sedimentación & Absetzbecken \\
\hline $\mathrm{N}+$ Adj. & 37 & cuerpo molturante & Mahlkörper \\
\hline NN-Kompositum & 5 & escurridera salva-gotas & Spritzschutz \\
\hline $\mathrm{N}+$ para $+\mathrm{N}$ & 4 & barbotina para colaje & Gießmasse \\
\hline
\end{tabular}

Macht man sich mit einer Reihe von Beispielen für jede Kategorie vertraut, ist unschwer zu erkennen, dass das Suffix '-dora' zur Benennung von Maschinen oder Maschineneinheiten verwendet wird, '-dor' dagegen eher für Maschinenteile. ' $\mathrm{N}+\mathrm{de}+\mathrm{N}$ ' und ' $\mathrm{N}+$ Adj.' scheinen bis $\mathrm{zu}$ einem gewissen Grad austauschbar zu sein, wobei durch Adjektive oft 
eine stärkere Implikation des Determinatums in der Aktion ausgedrückt wird (vgl. zum Beispiel cuerpo molturante $\rightarrow$ 'ein Körper, der mahlt', balsa de sedimentación $\rightarrow$ 'Becken, in dem das Absetzen stattfindet'). Die Präpositionalkonstruktion hat eine höhere Frequenz, was wohl darauf zurückzuführen ist, dass sich nicht zu jedem Begriff ein passendes Adjektiv ableiten lässt. Diese drei Bildungsformen machen das Gros der Benennungen aus. Komposita oder Konstruktionen mit der Präposition 'para' hingegen kommen nur auf 3,1 \% bzw. 2,5\% und haben somit nur eine geringe Bedeutung für die Bildung von Benennungen mit Finalrelation. Sie scheinen daher für die Neubildung von zielsprachlichen Benennungen nicht so gut geeignet zu sein. Die para-Konstruktion scheint aufgrund ihrer Nähe zu Paraphrasen mit bestimmtem Artikel (zum Vergleich: horno para la fusión de fritas) der Benennung einen schon fast umschreibenden Charakter zu geben.

Solche Kenntnisse über die sprachlichen Mittel, die in einer konkreten Fachsprache zur Erfüllung bestimmter Funktionen (hier der Ausdruck einer Finalrelation) eingesetzt werden, ihre annähernde Häufigkeitsverteilung sowie eventuelle Restriktionen können für den Übersetzer ein wichtiges Hilfsmittel für eine den Konventionen der Fachsprache angepasste Benennungsbildung sein.

\section{Konsequenzen für das Verhältnis Terminus - technischer Übersetzer}

In unserem Fazit zum Beitrag der neueren Ansätze der Terminologie für das Terminusverständnis des Übersetzers haben wir die Relativierung des Unterschieds zwischen Terminus und Wort unterstrichen. Wenn wir Termini damit in ihrem Status an Wörter annähern, dann bedeutet das jedoch nicht ein Weniger sondern ein Mehr. Es ist also keinesfalls gemeint, dass Termini nach Gutdünken behandelt und zum Beispiel paraphrasiert werden können. Die genannten textorientierten pragmatischen Überlegungen können erst dann sinnvoll eingesetzt werden, wenn die textspezifische Bedeutung ermittelt worden ist, wenn der Übersetzer sich also im Fachgebiet kundig gemacht und den Ausgangstext gründlich analysiert sowie mögliche zielsprachliche Termini recherchiert hat.

Bei diesen zusätzlichen Überlegungen des Übersetzers kommen die im Laufe unserer Darstellung immer wieder aufgetretenen Aspekte der Kommunikation und der Kognition ins Spiel. Eindeutigkeit entsteht vor allem im Text, und dabei gilt es die Verstehenstätigkeit des Lesers in die richtige Richtung zu leiten. Neben der Verwendung adäquater Terminologie ist es hier wichtig die für eine Sprache üblichen Textkonventionen (wie hier dargestellt im Bereich 'Kohäsion versus Redundanz') zu beachten. Zum anderen beschränkt sich die Bedeutung der Fachwortbildungskonventionen nicht auf die Schaffung neuer Benennungen für terminologische Lücken, sondern erstreckt sich auch auf den kritischen Umgang 
mit bestehenden (d. h. in Nachschlagewerken oder Paralleltexten dokumentierten) Äquivalenten, sei es durch die Auswahl der im Text adäquatesten Variante, sei es durch Ersetzung durch eine mehr oder weniger explizite Form, die den Textkonventionen der Zielsprache mehr entspricht. Es geht also um einen verantwortungsbewussten, aber auch selbstsicheren Umgang mit Termini, der auf ausreichender Information des Übersetzers bezüglich der Materie, der Terminologie und der Konventionen der Fachsprache beruht. Die terminologische Kompetenz des Übersetzers (und hiermit ist sowohl das Wissen um die Termini eines Fachgebiets als auch um die Methoden der Terminologie gemeint) muss eingebettet sein in fachsprachliche Kompetenz.

\section{Bibliographie}

Arntz, Reiner (1993). "Terminological Equivalence and Translation”. H. B. Sonneveld \& K. L. Loening (Hrsg.) (1993). Terminology. Applications in interdisciplinary communication. Amsterdam/Philadelphia: John Benjamins, 5-19.

Arntz, Reiner (2001). Fachbezogene Mehrsprachigkeit in Recht und Technik. Hildesheim: Georg Olms.

Cabré Castellví, M. Teresa (2003). "Theories of terminology: Their description, prescription and explanation". Terminology 9(2), 163-199.

Cabré, $\mathrm{M}^{\mathrm{a}}$ Teresa (1999). La Terminología: representación y comunicación: elementos para una teoría de base comunicativa y otros artículos. Barcelona: Institut Universitari de Lingüística Aplicada.

Dederding, Hans-Martin (1982). Wortbildung, Syntax, Text: Nominalkomposita und entsprechende syntaktische Strukturen in deutschen Patent- und Auslegeschriften. Erlangen: Palm \& Enke.

Dederding, Hans-Martin (1983). "Wortbildung und Text. Zur Textfunktion (TF) von Nominalkomposita (NK)". Zeitschrift für germanistische Linguistik 13(1), 49-64.

Dictionary of Chemical Engineering. English/French/German/Spanish (1992). Chichester: John Wiley \& Sons.

Drozd, L. \& W. Seibicke (1973). Deutsche Fach- und Wissenschaftssprache. Bestandsaufnahme - Theorie - Geschichte. Wiesbaden: Brandstetter.

Emsel, Martina (2001). "Por otra parte ohne Gegenstück. Semantische Aspekte in Lexikographie vs. Textvorkommen". G. Wotjak (Hrsg.) (2001). Studien zum romanisch-deutschen und innerromanischen Sprachvergleich. Frankfurt a. M.: Peter Lang, 591-603.

Felber, Helmut \& Gerhard Budin (1989). Terminologie in Theorie und Praxis. Tübingen: Narr.

Gamero Pérez, Silvia (2001). La traducción de textos técnicos. Barcelona: Ariel.

Gerzymisch-Arbogast, Heidrun (1996). Termini im Kontext. Verfahren zur Erschließung und Übersetzung der textspezifischen Bedeutung von fachlichen Ausdrücken. Tübingen: Narr.

Glosario europeo de la cerámica (1992). Faenza: Faenza editrice.

Gómez Pérez, Carmen (2001). La composición nominal alemana desde la perspectiva textual: El compuesto nominal como dificultad de traducción del alemán al español. Salamanca: Departamento de Traducción e Interpretación, Universidad de Salamanca. Dissertation. 
Göpferich, Susanne (1993). "Die translatorische Behandlung von Textsortenkonventionen in technischen Texten". Lebende Sprachen 38(2), 49-53.

Göpferich, Susanne (1995). Textsorten in Naturwissenschaften und Technik: Pragmatische Typologie, Kontrastierung, Translation. Tübingen: Narr.

Horn-Helf, Brigitte (1999). Technisches Übersetzen in Theorie und Praxis. Tübingen: Francke.

Jakob, Karlheinz (1991). Maschine, mentales Modell, Metapher: Studien zu Semantik und Geschichte der Techniksprache. Tübingen: Niemeyer.

Kaiser-Cooke, Michèle (1995). "Murder in the laboratory - Termhood and the culture gap". M. Snell-Hornby, Z. Jettmarová \& K. Kaindl (Hrsg.) (1995). Translation as Intercultural Communication. Amsterdam/Philadelphia: John Benjamins, 283-291.

Lakoff, George (1987). Women, Fire and Dangerous Things. What Categories Reveal about the Mind. Chicago: University of Chicago Press.

Mink, Hermann (1994). Technisches Fachwörterbuch, Deutsch - Spanisch. Bd. 1. 9. Auflage. Barcelona: Herder.

Oster, Ulrike (2003). Los términos de la cerámica en alemán y en español. Análisis semántico orientado a la traducción de los compuestos nominales alemanes. Dissertation. Universitat Jaume I, Castelló. Online unter http://www.tdx.cesca.es/TDX-1216103-131327/.

Oster, Ulrike (2004a). "Semantische Relationen in komplexen Fachbenen-nungen: Typen und Tendenzen im Spanischen und Deutschen". Estudios Filológicos Alemanes (5), 297-304.

Oster, Ulrike (2004b). "From relational schemas to subject-specific semantic relations: A two-step classification of compound terms". Annual Review of Cognitive Linguistics (2), 235-259.

Pearson, Jennifer (1998). Terms in Context. Amsterdam/Philadelphia: John Benjamins.

Rogers, Margaret (1999). "Translating Terms in Text: Holding on to Some Slippery Customers". G. Anderman \& M. Rogers (Hrsg.) (1999). Word, Text, Translation. Liber Amicorum for Peter Newmark. Clevedon, Buffalo: Multilingual Matters, 104-116.

Rogers, Margaret (2000). "Translating Technical Terms: Challenge or Pushover". The Linguist 39(4), 104-106.

Sager, Juan C. (1994). "Reflections on Terminological Translation Equivalents". Lebende Sprachen 39(2), 55-57.

Schmitt, Peter A. (1986). "Die Eindeutigkeit von Fachtexten: Bemerkungen zu einer Fiktion". M. Snell-Hornby (Hrsg.) (1986). Übersetzungswissenschaft - Eine Neuorientierung. Zur Integrierung von Theorie und Praxis. Tübingen: Francke.

Schmitt, Peter A. (1989). "Kulturspezifik von Technik-Texten. Ein translatorisches und terminographisches Problem". H. J. Vermeer (Hrsg.) (1989). Kulturspezifik des translatorischen Handelns. Heidelberg: IÜD, 49-88.

Schmitt, Peter A. (1999). Translation und Technik. Tübingen: Stauffenburg.

Schröder, Marianne (1978). "Über textverflechtende Wortbildungselemente". Deutsch als Fremdsprache 78, 285-92.

Schröder, Marianne (1983). "Zum Anteil von Wortbildungskonstruktionen an der Konstitution von Texten". Beiträge zur Erforschung der deutschen Sprache (3), 108-119.

Shreve, Gregory M. (1997). "Terminological Aspects of Text Production". S. E. Wright \& G. Budin (Hrsg.) (1997). Handbook of Terminology Management. Amsterdam/Philadelphia: John Benjamins, 772-787. 
Temmerman, Rita (2000). Towards New Ways of Terminology Description. Amsterdam/Philadelphia: John Benjamins.

Temmerman, Rita (2002). "Metaphorical models and the translator's approach to scientific texts". Linguistica Antverpiensia 2002(1), 211-226.

Thelen, Marcel (2002a). "Relations between terms: a cognitive approach. The interaction betweeen Terminology, Lexicology, Translation Studies and translation practice". Linguistica Antverpiensia 2002(1), 193-209.

Thelen, Marcel (2002b). "Translation Studies in the Year 2000: The State of the Art. Terminology in Theory and Practice". B. Lewandowska-Tomaszczyk \& M. Thelen (Hrsg.) (2002). Translation and Meaning. Part 6. Maastricht: Hogeschool Zuyd, Maastricht School of Translation and Interpretation, 21-39.

Varantola, Krista (1992). "Words, Terms and Translators". H. Tommola, et al. (Hrsg.) (1992). EURALEX '92. Proceedings I-II. Papers submitted to the 5th EURALEX International Congress on Lexicography in Tampere, Finland. Tampere: Tampereen Yliopisto, 121-128.

Weissenhofer, Peter (1992). "Zur Rolle der terminologischen Begriffslehre in der Translationswissenschaft”. M. Snell-Hornby, F. Pöchhacker \& K. Kaindl (Hrsg.) (1992). Translation Studies. An interdiscipline. Amsterdam/Philadelphia, 319-332.

Weissenhofer, Peter (1995). Conceptology in Terminology, Semantics and Word Formation. Wien: TermNet, International Network for Terminology.

Wladowa, E. W. (1975). "Okkasionelle Wortbildungen mit dem gleichen Stamm als Satz- und Textverflechtungsmittel". Textlinguistik (4), 71-87.

Wörterbuch der keramischen Fliesen- und Plattenindustrie. Deutsch-EnglishEspañol-Français-Italiano-Svenska (1970). Génova: CEC.

Wright, Sue Ellen \& Leland D. Wright (1997). "Terminology Management for Technical Translation”. S. E. Wright \& G. Budin (Hrsg.) (1997). Handbook of Terminology Management. Amsterdam/Philadelphia: John Benjamins, 147-159.

Wüster, Eugen (1959/60). "Das Worten der Welt, schaubildlich und terminologisch dargestellt". Sprachforum 3 (3/4), 183-204.

* Die Arbeit an diesem Artikel wurde durch ein Stipendium der Fundació Caixa Castelló-Bancaixa für einen Forschungsaufenthalt an der Universität Leipzig unterstützt. Für hilfreiche Kommentare möchte ich Dr. Martina Emsel danken.

${ }^{1}$ Unter technischer Übersetzung verstehen wir hier die Übersetzung von Texten aus den Fachgebieten der Technik und insbesondere von technischen Textsorten im Sinne von Gamero (2001). Terminus (oder auch Fachwort) wird wie in der deutschsprachigen Terminologie üblich als "Einheit aus Begriff und Benennung" verstanden. Geht es vorrangig um die Ausdrucksseite, verwenden wir auch Benennung.

${ }^{2}$ In einem allgemeinen technischen Wörterbuch (Mink 1994) sowie in fachspezifischen Wörterbüchern bzw. Datenbanken: Europäisches Glossar des Keramiksektors (1992), Dictionary of Chemical Engineering (1992), Wörterbuch der keramischen Fliesen- und Plattenindustrie (1970), Eurodicautom.

${ }^{3}$ Zur Begründung der Einordnung dieser Suffixe in der Fachsprache der Technik als Ausdruck der Final- und nicht der Aktionsrelation siehe Oster (2003: 396398). 
